\title{
CONTRIBUCIÓN AL CONOCIMIENTO DE LA FENOLOGÍA DEL CACTUS Neoraimondia arequipensis subsp. roseiflora (Werdermann \& Backeberg) Ostolaza (Cactaceae) EN EL VALLE DEL RÍO CHILLÓN, LIMA-PERÚ
}

\section{CONTRIBUTION TO THE KNOWLEDGE OF THE Neoraimondia arequipensis subsp. roseiflora (Werdermann \& Backeberg) Ostalaza (Cactaceae) CACTUS PHENOLOGY IN THE CHILLON RIVER VALLEY, LIMA, PERU}

\author{
Sidney Novoa ${ }^{1}$, Aldo Ceroni $^{1}$ y Consuelo Arellano ${ }^{2}$
}

\section{Resumen}

Se realizó un estudio sobre la fenología del cactus Neoraimondia arequipensis subsp. roseiflora en el cerro Umarcata, ubicado en la cuenca baja del río Chillón, Lima, Perú. Se marcaron en forma aleatoria 15 plantas adultas donde se evaluó el estado fenológico presente en diferentes épocas del año. Las observaciones fueron mensuales desde Octubre del 2003 hasta Septiembre del 2004. Mediante un Análisis de Correspondencias se estableció patrón hipotético de la secuencia de los estadios fenológicos de la planta. Los resultados indican la presencia de dos variables fenológicas bien marcadas, la Floración entre Noviembre y Abril y la Fructificación desde Mayo hasta Octubre. La fenología se presentó como un evento irregular, con ocho estadios. El estadio fenológico de botones florales (Noviembre) es el que se presenta en mayor proporción (59.44\%) durante todo el año, seguido del estadio fruto inmaduro (35.36\%) en Mayo y Septiembre, el de botones abortados (32.78\%) en Diciembre, Febrero y Marzo, el de frutos maduros (27.78\%) en Agosto, los de dispersión y floración total (12.78\%) en Julio y Abril, las flores marchitas $(10 \%)$ en Enero, los frutos apareciendo $(6.77 \%)$ en Octubre y el estadio vegetativo $(8.33 \%)$ en Junio.

Palabras clave: cactácea, fenofases, fenología, Neoraimondia arequipensis subsp. Roseiflora

\begin{abstract}
A phenology study of the Neoraimondia arequipensis subsp. roseiflora cactus was conducted at Umarcata hills, located in the lower Chillon river basin, Lima, Peru. Fifteen fully grown plants were randomly selected and marked in order to evaluate their phenological status through the different seasons over a year period. Observations were registered monthly from October 2003 to September 2004. Correspondence analysis was used to hypotesize about the pattern sequence of the cactus plant phenophases. Results indicate that two well marked phenological variables are present: Flowering between November and April, and fructification from May to October. Phenology appears as an irregular event, with eight phases. The phenophase of flowering buttons (November) was present more frequently (59.44\%) across the whole year; followed by inmature fruits $(35.36 \%)$ in May and September; aborted buttons $(32.78 \%)$ in December, February and March; mature fruit (27.78\%) in August; dispersion and total flowering (12.78\%) in July and April; dead flowers (10\%) in January; initiating fruits $(6.77 \%)$ in October and vegetative stage (8.33) in June.
\end{abstract}

Keywords: cactaceae, phenophases, phenology, Neoraimandia arequipensis subsp. roseiflora

\section{Introducción}

Las cactáceas cumplen un rol importante en los ecosistemas constituyendo un elemento esencial en el paisaje, gracias al sistema radicular amplio y superficial que forma una malla que interviene en los procesos de erosión y desertificación de los suelos y puesto que las raíces poseen pelos absorbentes caducos se constituyen como una fuente continua de materia orgánica que se incorpora al suelo (Magallanes, 1997). Los cactus interaccionan con diversos organismos en el ecosistema. Frente a las inclemencias del medio ambiente desarrollan estrechas relaciones con otras especies vegetales denominadas "plantas nodrizas" que ofrecen las condiciones de humedad y temperatura para el establecimiento de nuevas plántulas (Turner et al., 1966; Valiente et al., 1991a b; Valiente \& Ezcurra, 1991; Nobel, 1998; De Viana et al., 2000) y algunas veces crean las condiciones necesarias para el establecimiento de otros cactus (McAuliffe, 1984). Los tallos son la única fuente de fibra y agua para los animales silvestres en los ecosistemas desérticos (Nobel, 1998; Gibson \& Rundel, 2001; Márquez et al., 2003). Algunas aves utilizan a los tallos de los cactus como 
un inmejorable refugio frente a potenciales depredadores haciendo sus nidos sobre e incluso dentro de la planta (McAuliffe \& Hendricks, 1988; Rivera \& Rodriguez, 1998). Las flores y frutos son fuente de alimento de numerosas aves como colibríes y palomas, mamíferos como roedores y murciélagos, destacándose de estos grupos dos especies que se encuentran en peligro de extinción en el Perú como lo son la pava aliblanca (Penelope albipennis) y el murciélago longirostro peruano (Platalina genovesium) (Lerner et al., 2003; Sahley, 1996). Asimismo, una gran gama de insectos visitan estas plantas, de los cuales destacan las especies de los ordenes Lepidoptera, Diptera e Hymenoptera (Keopcke, 1973; Valiente et al., 1996; Valiente et al., 1997; Fleming \& Holland, 1998; Fleming et al., 2001; Godínez et al., 2002; Holland \& Fleming, 1999, 2002; Scobell \& Scout, 2002). Los estudios de fenología son de suma importancia no sólo en la comprensión de la dinámica de las comunidades vegetales, sino también como un indicador de la respuesta de estos organismos a las condiciones climáticas y edáficas de una zona en particular (Fournier, 1969). Los estados fenológicos de una especie pueden afectar a la planta a múltiples niveles, incluyendo los sucesos reproductivos individuales de la planta, interacciones con otros organismos (afectando la variación y abundancia espacio-temporal de polinizadores y dispersores), dinámicas poblacionales vegetales y el funcionamiento del ecosistema (Bronstein, 1995; McIntosh, 2002). Los estudios acerca de la fenología de las cactáceas son muy escasos, destacándose los trabajos recientes sobre la fenología reproductiva de tres especies simpátricas de cactus columnares, Stenocereus griseus (Haw.) Buxb, Pilosocereus repandus (L.) Backeberg y Pilosocereus lanuginosus (L.) Byles \& G.D. Rowley en la isla de Curaçao (Petit, 2001) y los de fenología floral en las dos especies de cactus: Ferocactus cylindraceus (Engelm.) Orcutt y Ferocactus wislizenii (Engelm.) Britton \& Rose en Tuczon, Arizona (McIntosh, 2002). Por otra parte, la fenología de cactáceas ha sido ampliamente considerada en otros procesos ecológicos tales como la polinización por murciélagos y polillas y dispersión de murciélagos (Sahley, 1996; Nassar, et al., 1997; Valiente et al., 1996, 1997; Fleming \& Holland, 1998; Rojas et al., 1999; Holland \& Fleming, 1999, 2002; De Viana et al., 2001; Molina et al., 2004, IbarraCerdeña et al., 2005). La especie Neoraimondia arequipensis subsp. roseiflora conocida como "gigantón" es un cactus de crecimiento arbustivo o arborescente como un candelabro, la subespecie roseiflora es nombrada así por el color de la flor que puede variar entre un rojizo a un rosado intenso a diferencia de la especie tipo que posee flores blancas. Su distribución abarca todos los valles y quebradas del departamento de Lima desde los Valles de Supe (límite Norte), Huaura, Chancay, Chillón, Rímac,
Lurín, Mala, Chilca, Omas, Cañete, y por el sur hasta Chincha y Pisco en el departamento de Ica. Altitudinalmente se distribuye entre los 500 y 2000 msnm. El único registro fenológico mencionado son las observaciones realizadas por Ostolaza (1982), acerca de la época de floración que se presenta de Julio a Noviembre. Por lo tanto, el presente trabajo tiene el objetivo de contribuir al conocimiento del comportamiento fenológico de Neoraimondia arequipensis subsp. roseiflora, en el cerro Umarcata, cuenca baja del río Chillón.

\section{Materiales y métodos}

Ubicación: El área de la investigación es en el cerro Umarcata, el cual se encuentra a una distancia de 6 kilómetros de Santa Rosa de Quives, a la altura del kilómetro 69 de la carretera Lima - Canta, a una altitud de 1260 msnm a $11^{\circ} 37^{\prime} 39^{\prime}$ ' L.S. y 7646'9', L.O. en el valle del río Chillón, Departamento de Lima, en la Provincia de Canta.

Vegetación en la zona de vida de la cuenca del río Chillón:

La cuenca baja del río Chillón está conformada por una sola zona de vida correspondiente al Matorral Desértico Subtropical (md-ST) el cual se ubica entre los 800 y $2100 \mathrm{msnm}$. Abarca un área de $2030 \mathrm{~km}^{2}$. El clima es árido y semicálido. La precipitación promedio anual es mayor a los $125 \mathrm{~mm}$ y la temperatura promedio anual es $18^{\circ} \mathrm{C}$. La vegetación natural está constituida en la parte más baja por un piso de cactáceas columnares y otra vegetación reducida. El monte ribereño está formado por: Salix humboldtiana, Acacia macracantha, Baccharis salicifolia, Arundo donax, Schinus molle y Caesalpinia spinosa. En el piso de cactáceas predominan: Neoraimondia arequipensis, Cereus amazonicus, Calymmanthium sp., Melocactus sp., Jatropha macrantha, Orthopterygium huancui y Viguiera sp. Estas plantas aumentan su densidad hacia los 1000 msnm (ONERN, 1976; Weberbauer, 1945).

Determinación del estado fenológico de

Neoraimondia arequipensis subsp. Roseiflora:

Se realizaron visitas mensuales desde Octubre del 2003 hasta Septiembre del 2004 en la que se marcaron 15 plantas adultas al azar donde se evaluaron el o los estados fenológicos presentes en diferentes épocas del año siguiendo la escala de Campbell (1970) citado por Rondón (1994) modificada para esta especie, considerando a los botones abortados como un estadio fenológico por presentarse en gran cantidad (Petit, 2001; McIntosh, 2002). Los datos sobre los estadios fenológicos fueron tomados bajo el criterio de presencia-ausencia utilizando un código de numeración que representa los ocho estadios reproductivos y un estadio vegetativo (Tabla 1; Figura 1). Posteriormente, se analizaron los datos obtenidos de las evaluaciones mensuales mediante un 
Análisis de Correspondencias, en su versión del programa estadístico R 2.0 (R Development Core Team, 2005) el cual se basa en una ordenación simultánea de filas y columnas de una matriz de datos (Greennacre, 1984; McCune \& Grace, 2002). A partir de una tabla de contingencia que en este caso fue la tabla que muestra el número de individuos que presentaron una o más de las distintas nueve fenofases durante los meses de evaluación, se creó una matriz de disimilitud transformada empleando la distancia Chi-cuadrado, la cual dará una mayor ponderación a los estados fenológicos cuyas abundancias hayan sido muy bajas dentro de la matriz original. Dicho análisis permitió establecer las posibles relaciones entre los estados fenológicos y los meses del año (tiempo) e identificar patrones de ocurrencia conjunta de estos estados fenológicos. Finalmente se construyeron fenogramas (Rondón, 1994) para ilustrar estos estados en el tiempo.

Tabla 1. Estados fenológicos de Neoraimondia arequipensis subsp. Roseiflora.

\begin{tabular}{|c|l|c|}
\hline \multirow{2}{*}{ FENOFASE } & \multicolumn{1}{c|}{$\begin{array}{c}\text { ESTADIO } \\
\text { FENOLÓGICO }\end{array}$} & $\begin{array}{c}\text { VALOR } \\
\text { FENOLÓGICO } \\
\text { ASIGNADO }\end{array}$ \\
\hline \multirow{3}{*}{ FLORACIÓN } & $\cdot$ Botones florales & 1 \\
& $\cdot$ Botones abortados & 2 \\
& $\cdot$ Floración total & 3 \\
& $\cdot$ Flores marchitas & 4 \\
\hline \multirow{2}{*}{ FRUCTIFICACIÓN } & $\cdot$ Frutos apareciendo & 5 \\
& $\cdot$ Frutos inmaduros & 6 \\
& $\cdot$ Frutos maduros & 7 \\
\hline DISPERSIÓN & $\cdot$ Frutos dehiscentes & 8 \\
\hline VEGETATIVO & \multicolumn{2}{c}{9} \\
\hline
\end{tabular}

Tabla 2. Número de individuos que presentaron cada una de las nueve fenofases durante Octubre 2003 - Setiembre 2004.

\begin{tabular}{|c|c|c|c|c|c|c|c|c|c|}
\hline \multirow{3}{*}{ MESES } & \multicolumn{9}{|c|}{ FENOFASES } \\
\hline & \multicolumn{4}{|c|}{ FLORACIÓN } & \multicolumn{3}{|c|}{ FRUCTIFICACIÓN } & \multirow{2}{*}{$\begin{array}{c}\text { DISP. } \\
8 \\
\end{array}$} & \multirow{2}{*}{$\begin{array}{c}\text { VEG. } \\
9\end{array}$} \\
\hline & 1 & 2 & 3 & 4 & 5 & 6 & 7 & & \\
\hline Enero & 11 & 6 & 5 & 3 & 0 & 3 & 3 & 0 & 0 \\
\hline Febrero & 9 & 7 & 2 & 0 & 0 & 2 & 1 & 0 & 0 \\
\hline Marzo & 11 & 7 & 0 & 1 & 0 & 2 & 1 & 0 & 0 \\
\hline Abril & 8 & 3 & 3 & 2 & 0 & 7 & 3 & 0 & 1 \\
\hline Mayo & 6 & 2 & 0 & 1 & 0 & 5 & 6 & 1 & 0 \\
\hline Junio & 2 & 0 & 3 & 0 & 3 & 9 & 6 & 3 & 1 \\
\hline Julio & 6 & 2 & 0 & 0 & 0 & 7 & 7 & 7 & 1 \\
\hline Agosto & 8 & 2 & 1 & 1 & 0 & 4 & 8 & 2 & 1 \\
\hline Septiembre & 9 & 7 & 0 & 1 & 0 & 4 & 6 & 1 & 1 \\
\hline Octubre & 11 & 5 & 1 & 2 & 4 & 2 & 0 & 0 & 1 \\
\hline Noviembre & 12 & 8 & 1 & 3 & 0 & 7 & 2 & 1 & 0 \\
\hline Diciembre & 13 & 8 & 4 & 0 & 0 & 6 & 0 & 0 & 0 \\
\hline TOTALES & 107 & 59 & 23 & 18 & 12 & 64 & 50 & 23 & 15 \\
\hline PROMEDIO & 8.92 & 4.92 & 1.92 & 1.50 & 1.00 & 5.33 & 4.17 & 1.92 & 1.25 \\
\hline$\%$ & 59.44 & 32.78 & 12.78 & 10.00 & 6.67 & 35.56 & 27.78 & 12.78 & 8.33 \\
\hline
\end{tabular}

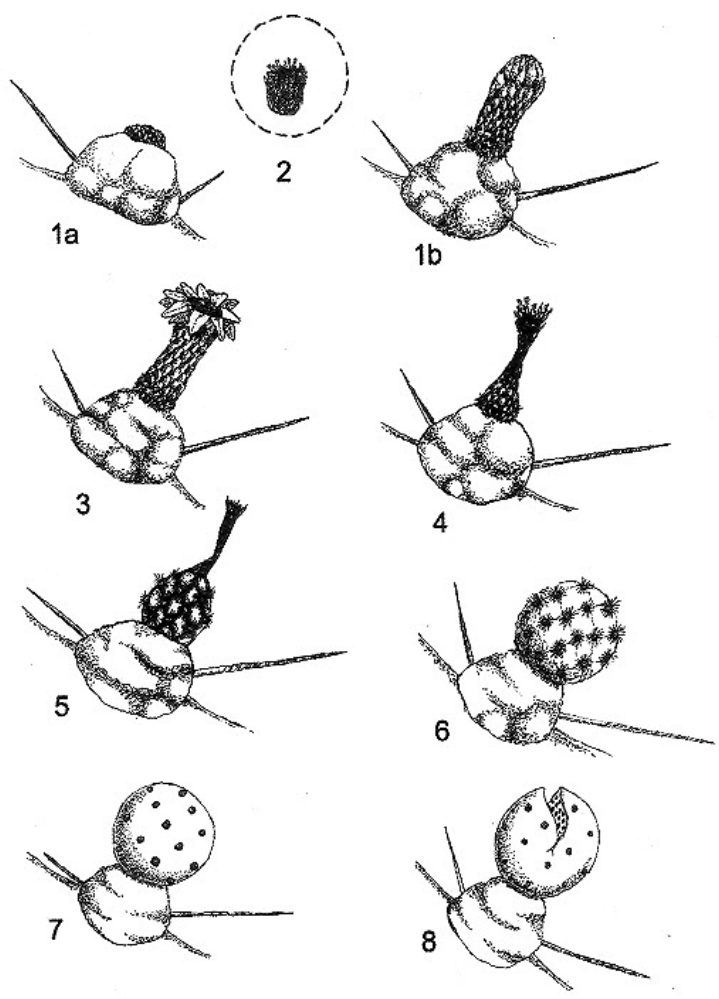

Figura 1. Estadios fenológicos de Neoraimondia arequipensis subsp. roseiflora. 1a y $\mathbf{1 b}$ botones florales, $\mathbf{2}$ botones abortados, $\mathbf{3}$ floración total, $\mathbf{4}$ flores marchitas, $\mathbf{5}$ frutos apareciendo, $\mathbf{6}$ frutos inmaduros o "verdes", 7 frutos maduros y $\mathbf{8}$ frutos dehiscentes (dispersión de semillas) (Dibujo:S.Novoa).

\section{RESULTADOS}

Determinación del estado fenológico de Neoraimondia arequipensis subsp. roseiflora

Se encontraron ocho estadios fenológicos de los cuales el estadio botón floral (59.44\%) fue el más abundante, seguido del estadio fruto inmaduro $(35.36 \%)$ botones abortados (32.78\%), frutos maduros $(27.78 \%)$, dispersión y floración total $(12.78 \%)$, flores marchitas $(10 \%)$, frutos apareciendo $(6.77 \%)$ y vegetativo $(8.33 \%)$ (Tabla 2; Figuras 2 y 3). Debido a la irregularidad - aparente - de su fenología, en la que una sola planta puede presentar hasta cuatro estadios diferentes (Tabla 3) en un solo mes y que muchos de estos estadios se repetían con igual magnitud en diversos meses del año se procedió a analizar los datos a través de un Análisis de Correspondencias. Se constató la presencia de dos variables fenológicas bien marcadas a lo largo del periodo de evaluación, floración y fructificación (Figuras 2 y 3). Sin embargo, cada una de estas se encuentra formada por cuatro estadios fenológicos que se hacen presentes a lo largo del año, que en el caso 
específico de este cactus, no permite establecer un patrón secuencial de los mismos. La floración se desarrolla en los meses de Noviembre a Abril y la fructificación en Mayo y Octubre. El patrón hipotético propuesto sobre el patrón secuencial de la fenología de $N$. arequipensis es: a Noviembre le corresponde el estadio botón floral; a Diciembre le corresponde el de botón abortado al igual que a Febrero y Marzo; al mes de Enero le corresponde el estadio fenológico de flor marchita; al mes de Abril, el estadio floración total; Mayo y Septiembre, el estadio fenológico de fruto inmaduro o - verde -; Agosto con fruto maduro; Julio con dispersión de semillas mediante la dehiscencia del fruto; y Octubre con los frutos apareciendo. Finalmente el mes de Junio queda como el correspondiente al estadio vegetativo (Figura 4). Un evento tan desigual como lo es la fenología de esta especie puede ser confundido y equivocado especialmente cuando se tiene un tamaño de muestra pequeño o se opta por el conteo de las estructuras reproductivas, lo que puede llevar a pensar que no existiría una secuencia clara de los estadios fenológicos. Cabe resaltar que lo más notorio de esta secuencia estacional fue la constante producción de botones florales en gran parte del año, que podría hallar su explicación en base a sus características anatómicas. $N$. arequipensis es un cactus que se ramifica desde la base, sus tallos no son articulados lo cual significa que a diferencia de otras especies de cactáceas, su reproducción vegetativa es muy poco probable. Esta característica particular obliga a la planta a tener que reproducirse sexualmente y generar nuevas plántulas mediante la germinación de sus semillas. Otra característica a tomar en cuenta es el crecimiento elongado de las areolas, las plantas siempre muestran el dilema de distribuir los escasos recursos entre la producción de estructuras florales o estructuras vegetativas (crecimiento

Tabla 3. Valores fenológicos asignados de Neoraimondia arequipensis subsp. roseiflora durante Octubre 2003 - Setiembre 2004.

\begin{tabular}{|c|c|c|c|c|c|c|c|c|c|c|c|c|}
\hline & \multicolumn{12}{|c|}{ MESES } \\
\hline INDIVIDUO & ENE & FEB & MAR & $\mathrm{ABR}$ & MAY & JUN & JUL & $\mathrm{AGO}$ & SEP & OCT & NOV & DIC \\
\hline 1 & 2 & 2 & 4 & 1,4 & 1,4 & 6 & 2,8 & 1,4 & $1,7,8$ & 2,4 & 2,4 & 2 \\
\hline 2 & 3,4 & 2 & 1,2 & 6 & 6 & 6,7 & 1,8 & 1,2 & $1,2,6$ & 2,4 & $1,2,4$ & 1,2 \\
\hline 3 & 1,2 & 2 & 2 & 1 & 1,6 & 3,6 & 2 & 2 & 2,6 & 1,2 & 1,6 & 1,2 \\
\hline 4 & 1,7 & 1,2 & 1 & 9 & 1,7 & 7,8 & 1 & 1 & 7 & 1 & 1 & 1,6 \\
\hline 5 & $1,3,6$ & 1,2 & 1,2 & $6,7,1$ & 6,7 & 3,7 & $6,7,8$ & $3,6,7$ & 2,7 & 1,6 & $1,3,7,6$ & 1 \\
\hline 6 & 1,4 & 9 & 2 & $1,3,6$ & 7 & 7,8 & 1 & 2,6 & 1,2 & 1,2 & 1,6 & 1,2 \\
\hline 7 & 2 & 2 & 1,6 & 3,4 & 1 & 6,7 & $1,7,6$ & $1,6,7$ & 1,2 & 1,5 & 1,6 & 1,3 \\
\hline 8 & 1,2 & 3,6 & 7 & 6,7 & 7 & 1 & 7 & 7 & 1 & 1,5 & 6,7 & $1,3,6$ \\
\hline 9 & 1,3 & 1 & 1 & 1 & 1 & 5,6 & 7,8 & 7,8 & 1,2 & 1 & $1,2,4$ & $1,2,6$ \\
\hline 10 & 1,7 & 1 & 1,2 & 1,2 & 6 & 5,6 & $6,7,8$ & 7 & $1,2,7$ & 1,5 & 1,2 & $1,3,6$ \\
\hline 11 & $1,2,7$ & 1,7 & 1,2 & 6,7 & 7,8 & 3,7 & $6,7,8$ & 1,7 & 1,7 & 1,3 & $1,2,6$ & $1,3,6$ \\
\hline 12 & 1,6 & 1 & 1 & 1,2 & 2 & 5,6 & 1,6 & $1,7,8$ & $1,4,6$ & 1,6 & 1,8 & $1,6,2$ \\
\hline 13 & 1,6 & 1,6 & 1 & 6 & 9 & 9 & 9 & 9 & 9 & 9 & 2 & 1 \\
\hline 14 & 3,4 & 1,3 & 1,6 & 6,3 & 6,7 & 8,6 & $6,7,8$ & $1,6,7$ & $1,6,7$ & 1,5 & $1,2,6$ & 1,2 \\
\hline 15 & $1,2,3$ & 1,2 & 1,2 & 1,2 & 1 & 1,6 & 1,6 & 1,2 & 1 & 2 & 1,2 & 2 \\
\hline
\end{tabular}
del tallo de las ramas). La elongación de las areolas se muestra como una buena alternativa para reducir el costo de producción de flores al mínimo, además de que cada areola puede producir una o más flores año tras año a diferencia de la mayoría de los cactus que producen una sola flor al año (Mauseth \& Kiesling, 1997; Mauseth et al., 2002). Esto último, sumado al número de ramas (que puede variar entre 1 y más de 20) y estas a su vez poseen areolas generadoras de estructuras reproductivas en potencia, hacen que el evento de producción de botones florales sea masivo y significativo a lo largo del año.

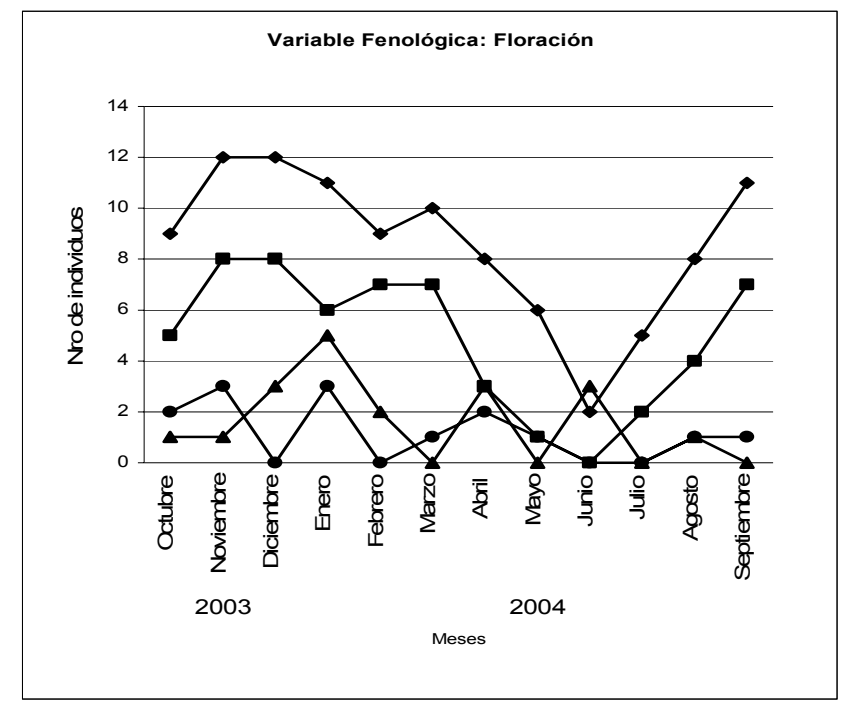

Figura 2. Fenograma de las etapas de la floración de Neoraimondia arequipensis subsp. roseiflora. Octubre 2003 - Setiembre 2004. (Botones florales $\rightarrow-$, Botones abortados - , Floración total $\rightarrow$, Flores marchitas $\rightarrow-$ ).

\section{Agradecimientos}

Los autores expresan su agradecimiento a los estudiantes de Biología de la UNALM que colaboraron en la toma de datos. Al Dr. Alfonso Valiente Banuet de la Universidad Autónoma de México (UNAM) y al Dr. Carlos Ostolaza de la Sociedad Peruana de Cactus y Suculentas (SPECS), por facilitarnos parte de la bibliografía. Esta investigación fue financiada por el Fondo Especial de Desarrollo Universitario (FEDU) de la Universidad Nacional Agraria La Molina. 


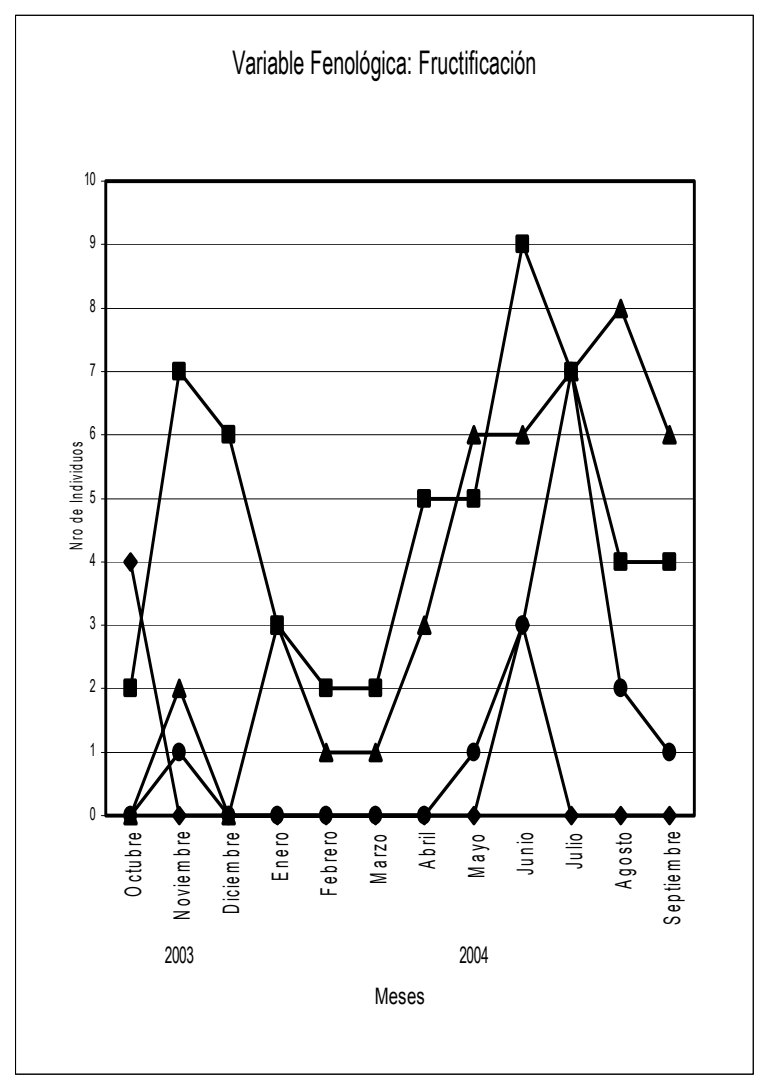

Figura 3. Fenograma de las etapas de la fructificación de Neoraimondia arequipensis subsp. roseiflora. Octubre 2003 - Setiembre 2004. (Frutos apareciendo $\rightarrow-$, Frutos inmaduros - , Frutos maduros $\rightarrow$, Dispersión $\rightarrow-$ )

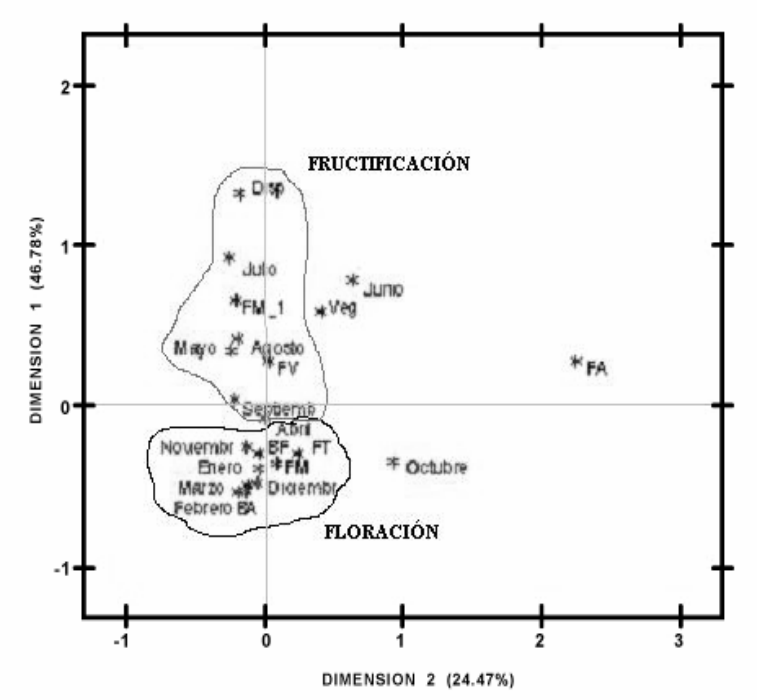

Figura 4. Resultado del Análisis de Correspondencia entre las nueve fenofases y los meses Octubre 2003 a Setiembre 2004.

\section{Literatura citada}

Bronstein J.L. 1995. The plant-pollinator landscape. In: Hansson L., Fahrig L. and Merriam G. (eds), Mosaic Landscapes and Ecological Processes. Chapman \& Hall, London.

De Viana M., Sühring S. \& Manly B. 2000. Application of randomization methods to study the association of Trichocereus pasacana (Cactaceae) With Potential Nurse Plants. Plant Ecology. 156(2): 1-5.

De Viana M., Ortega B., Saravia M. \& Schlumpberger B. 2001. Biología floral y polinizadores de Trichocereus pasacana (Cactaceae) en el Parque Nacional Los Cardones, Argentina. Revista de Biología Tropical. 49(1): 279-285.

Fleming T. \& Holland J. 1998. The evolution of obligate pollination mutualisms: senita cactus and senita moth. Oecologia. 114: 368-375.

Fleming T., Sahley C., Holland J., Nassar J. \& Hamrick J. 2001. Sonoran desert columnar cacti and the evolution of generalized pollination systems. Ecological Monographs. 71(4): 511-530.

Fournier L. 1969. Estudio preliminar sobre la floración en el roble de la sabana, Tabebuia pentaphylla (L.) Helmst. Revista de Biología Tropical. 15(2): 259-267.

Gibson A. \& Rundel P. 2001. Browningia candelaris in the Andean Pre-puna of northern Chile. Cactus \& Succulent Journal (U.S.). 73 (1): 27-33.

Godínez H., Valiente A. \& Rojas A. 2002. The role of seed dispersers in the population dynamics of the columnar cactus Neobuxbaumia tetetzo. Ecology. 83(9): 2617-2629.

Greenacre M. 1984. Theory and Applications of Correspondence Analysis. Academic Press, London.

Holland J. \& Fleming T. 1999. Mutualistic interactions between Upiga virescens (Pyralidae), a pollinating seedconsumer, and Lophocereus schottii (Cactaceae). Ecology. 80(6): 2074-2084.

2002. Co-pollinators and specialization in the pollinating seed-consumer mutualism between senita cacti and senita moths. Oecología. 133: 534-540.

Ibarra-Cerdeña C., Iñiguez-Dávalos L. \& Sánchez-Cordero V. 2005. Pollination ecology of Stenocereus queretaroensis (Cactaceae) a chiropterophilous columnar cactus, in a tropical dry forest of México. American Journal of Botany. 92(3): 503-509.

Keopcke H. 1973. Die Lebensformen Band I. Goecke \& Evers- Krefeld.

Lerner T., Ceroni A. \& González C. 2003. Etnobotánica de la Comunidad Campesina ". Santa Catalina de Chongoyape ". Ecología Aplicada. 2(1): 14-20.

Magallanes C. 1997. Cactáceas de la Provincia de Huamanga. Tesis para obtener el Título de Magíster Scientae. UNALM. Lima, Perú.

Márquez C., Vargas H., Snell H., Mauchamp A., Gibas J. \& Tapia W. 2003. ¿ Por que tan pocas Opuntias en la Isla Española-Galápagos?. Ecología Aplicada. 2(1): 2129.

Mauseth J. \& Kiesling R. 1997. Comparative anatomy of Neoraimondia reoseiflora and Neocardenasia herzogiana (Cactaceae). Haseltonia. 5: 37-49.

Mauseth J., Kiesling R. \& Ostolaza C. 2002. A Cactus Odissey. Journeys in the wilds of Bolivia, Perú and Argentina. Timber Press. Oregon. USA. 
McAuliffe J. 1984. Prey refugia on the distributions of two sonoran desert cacti. Oecología. 65: 82-85.

McAuliffe J. \& Hendriks P. 1988. Determinants of the vertical distributions of woodpecker nest cavities in the Sahuaro cactus. The Condor. 90: 791-801.

McCune B. \& Grace J. 2002. Analysis of Ecological Communities. MjM Software Design.Gleneden Beach. Oregon.

McIntosh M. 2002. Flowering phenology and reproductive output in two sister species of Ferocactus (Cactaceae) Plant Ecology. 159: 1-13.

Molina F., Rojas A., Fleming T. \& Valiente A. 2004 Pollination biology of the columnar cactus Pachycereus pecten-aboriginum in north-western México. Journal of Arid Environments. 56: 117-127.

Nassar J., Ramírez N. \& Linares O. 1997. Comparative pollination biology of venezuelan columnar cacti and the role of nectar-feeding bats in their sexual reproduction. American Journal of Botany. 84(8): 919-927.

Nobel P. 1998. Los Incomparables Agaves y Cactos. Ed. Trillas. México.

Oficina Nacional de Evaluación de Recursos Naturales (ONERN).1976. Mapa Ecológico del Perú: Guía Explicativa. Lima, Perú.

Ostolaza C. 1982. Neoraimondia roseiflora (Werd \& Backbg) Backbg. Boletín de Lima. 19: 89-93.

Petit S. 2001. The reproductive phenology of three sympatric species of columnar cacti on Curaçao. Journal of Arid Enviroments. (49): 521-531.

R Development Core Team. 2005. R: A language and environment for statistical computing. R Foundation for Statistical Computing, Vienna, Austria. ISBN 3-90005107-0, URL http://www.R-project.org.

Rivera L. \& Rodríguez R. 1998. Breeding biology of the crested caracara in the cape region of Baja California, México. Journal of Field Ornithology. 69(2): 160-168.

Rojas A., Valiente A., Arizmendi M., Alcántara A. \& Arita H. 1999. Seasonal distribution of the long-nosed bat (Leptonicteris curosoae) in the North America: does a generalized migration pattern really exist?. Journal of Biogeography. 26: 1065-1077.
Rondón J. 1994. Sinopsis de las principales metodologías aplicadas a los estudios fenológicos de los árboles tropicales. Revista Forestal Latinoamericana. 14: 5-32.

Sahley C. 1996. Bat and huming bird pollination of an autotetraploid columnar cactus, Weberbanerocereus webwerbaueri (Cactaceae). American Journal of Botany. 83(10): 1329-1336.

Scobell S. \& Scout P. 2002. Visitor and floral traits of huming birds-adapted cactus (Echinocereus coccineus) show only minor variation along an elevational gradient. The American Midland Naturalist. 17(1): 1-15.

Turner R., Alcorn S., Olin G. \& Booth J. 1966. The influence of shade, soil, and water on Saguaro seedling establishment. Botanical Gazette. 127(2-3): 95-102.

Valiente A. \& Ezcurra E. 1991. Shade as a cause of association between the cactus Neobuxbaumia tetetzo and the nurse plant Mimosa luisiana in the Tehuacan Valley. Mexico. Journal of Ecology. 79: 961-971.

Valiente A., Vite F. \& Zavala A. 1991a. Interaction between the cactus Neobuxbaumia tetetzo and the nurse shrub Mimosa luisiana. Journal of Vegetation Science. 2: 1114.

Valiente A., Bolongaro A., Briones O., Ezcurra E., Rosas M., Núñez H., Barnard G. \& Vázquez E. 1991b. Relatonships between cacti and nurse shrubs in a semiarid evironment in Central México. Journal of Vegetation Science. 2: 15-20.

Valiente A., Del Coro M., Rojas A. \& Dominguez L. 1996. Ecological relationships between columnar cacti and nectar-feeding bats in México. Journal of Tropical. Ecology. 12(1): 103-119.

Valiente A., Rojas A., Del Coro M. \& Davila P. 1997. Pollination Biology of two columnar cacti (Neubuxbaumia mezcalaensis and Neobuxbaumia macrocephala) in the Tehuacan Valley, central Mexico. American Journal of Botany. 84(4): 452-455.

Weberbauer A. 1945. El mundo vegetal de los Andes Peruanos: Estudio Fitogeográfico. Estación Experimental Agrícola La Molina. Lima, Perú.

1 Jardín Botánico “Octavio Velarde Núñez”. Departamento Académico de Biología. Universidad Nacional Agraria La Molina. Av. La Molina s/n, Apartado Postal 12056, Lima100. Perú, 2000084@lamolina.edu.pe, aceroni@lamolina.edu.pe

2 Gabinete de Investigación en Ecología Cuantitativa. Departamento Académico de Biología. Universidad Nacional Agraria La Molina, carellano@lamolina.edu.pe 